Article

\title{
Multi-Fidelity Multi-Objective Efficient Global Optimization Applied to Airfoil Design Problems
}

\author{
Atthaphon Ariyarit ${ }^{*,+}$ (1) and Masahiro Kanazaki \\ Department of Aerospace Engineering, Graduate School of System Design, Tokyo Metropolitan University, \\ Hino-shi, Tokyo 191-0065, Japan; kana@tmu.ac.jp \\ * Correspondence: atthaphon@msn.com; Tel.: +66-86-972-5642 \\ + These authors contributed equally to this work.
}

Received: 30 October 2017; Accepted: 15 December 2017; Published: 18 December 2017

\begin{abstract}
In this study, efficient global optimization (EGO) with a multi-fidelity hybrid surrogate model for multi-objective optimization is proposed to solve multi-objective real-world design problems. In the proposed approach, a design exploration is carried out assisted by surrogate models, which are constructed by adding a local deviation estimated by the kriging method and a global model approximated by a radial basis function. An expected hypervolume improvement is then computed on the basis of the model uncertainty to determine additional samples that could improve the model accuracy. In the investigation, the proposed approach is applied to two-objective and three-objective optimization test functions. Then, it is applied to aerodynamic airfoil design optimization with two objective functions, namely minimization of aerodynamic drag and maximization of airfoil thickness at the trailing edge. Finally, the proposed method is applied to aerodynamic airfoil design optimization with three objective functions, namely minimization of aerodynamic drag at cruising speed, maximization of airfoil thickness at the trialing edge and maximization of lift at low speed assuming a landing attitude. XFOILis used to investigate the low-fidelity aerodynamic force, and a Reynolds-averaged Navier-Stokes simulation is applied for high-fidelity aerodynamics in conjunction with a high-cost approach. For comparison, multi-objective optimization is carried out using a kriging model only with a high-fidelity solver (single fidelity). The design results indicate that the non-dominated solutions of the proposed method achieve greater data diversity than the optimal solutions of the kriging method. Moreover, the proposed method gives a smaller error than the kriging method.
\end{abstract}

Keywords: multi-fidelity optimization; efficient global optimization; multi-objective optimization; airfoil design

\section{Introduction}

A high-cost computation function is required to solve aerodynamic design problems. In addition, real-world design problems often involve several objective functions [1,2]. For example, in aircraft design, it is necessary for a designer to account for not only the performance at a specific cruise condition, but also the performances at all operating speeds, including those during take-off and landing. Owing to these problems, several researchers have explored methods to reduce the computational costs of design optimization algorithms for multi- or many-objective optimization problems.

In aerodynamic evaluation, it is possible to select various physical computation models to solve a design problem [3]. For example, forces around an airfoil can be evaluated by a potential equation as a low-level computation [4]. Moreover, the Navier-Stokes equation can be employed as a high-level computation [5]. Given the advantage of the above-mentioned approach, a multi-fidelity approach combines two-fidelity data for optimization in order to improve the efficiency of the optimization 
process. Thus, approaches that include a multi-fidelity surrogate model have been widely studied [6-8] in aerospace engineering. Multi-fidelity methods for single-objective optimization based on the error estimation of response surfaces have been successfully applied to design low-boom supersonic jets [6]. Because the response surfaces [9] use simple concepts to construct the function, the multi-fidelity function based on the response surface is easy to construct. However, such methods are often associated with low accuracy because the response surface model cannot be optimized in situations involving an extremely small number of data points. A co-kriging model $[7,8]$ has been extensively applied to combine multi-fidelity functions. In addition, it has been employed to solve an optimal single-objective airfoil design problem [7]. This method is beneficial for predicting a complicated landscape function. However, it is less optimal in predicting a smooth landscape function. Rethore proposed a multi-fidelity single-objective optimization process for wind turbine design $[10,11]$. This optimization process begins with the location of an optimal point of a low-fidelity function by using a genetic algorithm (GA). Then, an optimization with a high-fidelity function is performed using a gradient-based method. The optimum point of the low-fidelity function is used as a starting point. This method is beneficial, as it uses a basic optimization tool to solve the multi-fidelity optimization problem. However, it could fail to find an optimum high-fidelity function if the error between the low-fidelity and high-fidelity functions is large. Because the gradient-based method required many evolution functions to solve multi-objective optimization problems, the multi-fidelity optimization based on evolutionary computation combined with the gradient method would not reduce the computation time of the high-fidelity function. Thus, multi-fidelity approaches are expected to improve the efficiency.

An approach to multi-fidelity/multi-objective optimization involves a model reduction technique $[12,13]$. This technique has been applied to helicopter rotor blade design and airfoil design $[13,14]$. The application of this technique reduces the design parameter space for defining the possible design ranges based on a low-fidelity function. A high-fidelity function is then used to find the optimum design in the primary defined design range. The high-fidelity function is sampled by selecting the preferred design points, where the blade shows improved performance with respect to the baseline design, in order to ensure good diversity of the sample data, in addition to the initial point that is generated with the primary defined design range. However, this approach has the potential to obtain an unexpected optimal solution outside the parameter space given that the design ranges of the low-fidelity function are not always appropriate for the high-fidelity function. Fusi [15] used GA to find the optimum solution of low-fidelity data and selected the interesting points of the optimum solution using the low-fidelity data to find the optimum point of high-fidelity data of a hovering rotor airfoil design.

Efficient global optimization (EGO) [16] is a widely-used method that consists of kriging-model-based explorations. EGO involves additional sampling-procedure-based expected improvements (EIs) for improving the model accuracy. The EIs are defined for single-objective optimization. The expected hypervolume improvement (EHVI) algorithm $[17,18]$ has been proposed to improve the non-dominated front defining the expectations of hypervolume improvements (HVI); it considers the model uncertainty, as well as the EIs. However, existing studies have not investigated the applicability of EHVI to multi-fidelity techniques. In this article, a multi-objective optimization process involving multi-fidelity/multi-objective EGO is proposed and investigated through the solutions of test functions and airfoil design problems. With respect to airfoil design problems, a low-fidelity function is used to construct a global model that can provide the global landscape of the function. Further, a high-fidelity function is used to evaluate local deviations. The global model, constructed by a radial basis function (RBF) $[19,20]$ based on a database, is evaluated using low-fidelity models. Local variances are predicted using a correlation term of the kriging method. The study involves airfoil design problems with two and three objectives. The results of the optimization are compared with those of an ordinary kriging-based EHVI involving a single-fidelity approach. 


\section{Surrogate Models for Design Optimization}

\subsection{Kriging Method}

An ordinary kriging method [21] predicts the unknown function $\hat{y}(\mathbf{x})$ as:

$$
\hat{y}(\mathbf{x})=\mu+\varepsilon(\mathbf{x}),
$$

where $\mu$ is global model and $\varepsilon(\mathbf{x})$ is a local deviation. The sample points $\mathbf{x}$ are interpolated with Gaussian random function. The correlation between $Z\left(x_{i}\right)$ and $Z\left(x_{j}\right)$ is related to the distance between the two corresponding point $x_{i}$ and $x_{j}$. A local deviation $\varepsilon(\mathbf{x})$ is expressed as:

$$
d\left(\mathbf{x}_{i}, \mathbf{x}_{j}\right)=\sum_{k=1}^{n} \theta^{k}\left|x_{i}^{k}-x_{j}^{k}\right|^{2}
$$

where $\theta^{k}\left(0 \leq \theta^{k} \leq \infty\right)$ is the $k$-th element of the correlation vector parameter and $n$ is the number of the sample points. The correlation between the points $x_{i}$ and $x_{j}$ is defined as:

$$
\operatorname{Corr}\left[Z\left(x_{i}\right), Z\left(x_{j}\right)\right]=\exp \left[-d\left(x_{i}, x_{j}\right)\right] .
$$

The kriging prediction can be expressed as:

$$
\hat{y}(\mathbf{x})=\mu+\mathbf{r}^{\mathrm{T}} \mathbf{R}^{-1}(\mathbf{F}-\hat{\mu}),
$$

where $Z\left(x_{i}\right)$ represents a local deviation from the global model [22], $\mathbf{F}=$ $\left[f\left(x_{1}\right), f\left(x_{2}\right), f\left(x_{3}\right), \ldots, f\left(x_{n}\right)\right]^{\mathrm{T}}$ is the value of the evaluated function at $\mathbf{X}=\left\{x_{1}, x_{2}, x_{3}, \ldots, x_{n}\right\}$, $\mathbf{R}$ denotes the $n \times n$ matrix whose $(i, j)$ entry is $\operatorname{Corr}\left[Z\left(x_{i}\right), Z\left(x_{j}\right)\right]$ and $\mathbf{r}$ is the vector $i$-th element:

$$
r_{i}(\mathbf{x})=\operatorname{Corr}\left[Z(x), Z\left(x^{i}\right)\right] .
$$

$\mu$ is assumed to be constant in the original kriging model, and $\hat{\mu}$ is given by:

$$
\hat{\mu}=[\mu, \mu, \mu, \ldots, \mu]^{\mathrm{T}},
$$

where $\mu$ is defined as:

$$
\mu=\frac{\mathbf{1}^{\mathrm{T}} \mathbf{R}^{-1} \mathbf{F}}{\mathbf{1}^{\mathrm{T}} \mathbf{R}^{-1} \mathbf{1}} .
$$

The unknown parameter, $\theta$, for the kriging model can be estimated via maximum likelihood estimation (MLE):

$$
\operatorname{Ln}\left(\mu, \sigma^{2}, \theta\right)=-\frac{n}{2} \ln \left(\sigma^{2}\right)-\frac{1}{2} \ln (|\mathbf{R}|) .
$$

MLE is an $m$-dimensional unconstrained nonlinear optimization problem. In this article, a GA [23] is used to solve this problem. For a given $\theta, \sigma^{2}$ can be defined as:

$$
\sigma^{2}=\frac{(\mathbf{F}-\hat{\mu})^{\mathrm{T}} \mathbf{R}^{-1}(\mathbf{F}-\hat{\mu})}{n} .
$$

The mean square error $s^{2}(\mathbf{x})$ at a point $\mathbf{x}$ of this function can be calculated using the following equation:

$$
s^{2}(\mathbf{x})=\sigma\left[1-\mathbf{r}^{\mathrm{T}} \mathbf{R}^{-1} \mathbf{r}+\frac{1-\mathbf{1}^{\mathrm{T}} \mathbf{R}^{-1} \mathbf{F}}{\mathbf{1}^{\mathrm{T}} \mathbf{R}^{-1} \mathbf{1}}\right],
$$

where 1 denotes an $n$-dimensional unit vector. 


\subsection{Hybrid Surrogate Model for Multi-Fidelity Approach}

In this section, the hybrid surrogate model (Figure 1) is proposed for the multi-fidelity approach. The proposed approach constructed the local deviation estimated by the kriging method and the global model approximated by the RBF. It employs an RBF to represent the global model, $\mu+f r(\mathbf{x})$, based on a dataset obtained from low-fidelity evaluation. The proposed approach combines the kriging method and the RBF by the following equation:

$$
\hat{y}(\mathbf{x})=[\mu+f r(\mathbf{x})]+\mathbf{r}^{\mathrm{T}} \mathbf{R}^{-1}\left(\mathbf{F}_{\mathbf{h}}-\hat{\mu}-\mathbf{F R}\right) .
$$

The local deviations $\mathbf{r}^{\mathrm{T}} \mathbf{R}^{-1}\left(\mathbf{F}_{\mathrm{h}}-\hat{\mu}-\mathbf{F R}\right)$ are evaluated on the basis of a high-fidelity dataset using the kriging method, where $\mathbf{F}_{\mathrm{h}}=\left[f_{\mathrm{h}}\left(x_{1}\right), f_{\mathrm{h}}\left(x_{2}\right), f_{\mathrm{h}}\left(x_{3}\right), \ldots, f_{\mathrm{h}}\left(x_{n}\right)\right]^{\mathrm{T}}$ is the value of the high-fidelity function at $\mathbf{x}=\left\{x_{1}, x_{2}, x_{3}, \ldots, x_{n}\right\} ; \mathbf{F R}=\left[f r\left(x_{1}\right), f r\left(x_{2}\right), f r\left(x_{3}\right), \ldots, f r\left(x_{n}\right)\right]^{\mathrm{T}}, f r(\mathbf{x})$ the function predicted from the low-fidelity data that predicted by RBF, can be expressed by (12):

$$
f r(\mathbf{x})=a_{0}+a_{1} f_{1}(\mathbf{x}),
$$

where $f_{1}(\mathbf{x})$ is a function predicted by an $\operatorname{RBF}[19,20]$ using low-fidelity data and $a_{0}$ and $a_{1}$ are correlation terms between the low-fidelity data and the high-fidelity data. Further, $\sigma^{2}$ can be defined as:

$$
\sigma^{2}=\frac{(\mathbf{F}-\hat{\mu}-\mathbf{F R})^{\mathrm{T}} \mathbf{R}^{-1}(\mathbf{F}-\hat{\mu}-\mathbf{F R})}{n} .
$$

The unknown parameters $\left(\theta, a_{0}\right.$ and $\left.a_{1}\right)$ for the hybrid surrogate multi-fidelity model can be estimated by MLE, as expressed by (8).

The RBF is used to approximate the global model of the hybrid surrogate-model. An RBF is used to predict the low-fidelity function $\left(f_{1}(\mathbf{x})\right)$ by:

$$
f_{1}(\mathbf{x})=\sum_{m=1}^{n} w_{m} \Phi\left(\mathbf{x}-\mathbf{x}_{m}\right)
$$

where $\Phi(\mathbf{x})$ is an RBF, $\mathbf{x}_{m}$ is a sample point and $w_{m}(m=1,2,3, \ldots, n)$ is a weighting function. A multi-quadratic function is applied as an RBF in this study. The weighting function $\mathbf{w}=\left[w_{1}, w_{2}, w_{3}, \ldots, w_{n}\right]^{\mathrm{T}}$ is determined from the interpolation conditions:

$$
A \mathbf{w}=\mathbf{F}
$$

where $A=\left(\begin{array}{cccc}a_{1,1} & a_{1,2} & \cdots & a_{1, j} \\ a_{2,1} & a_{2,2} & \cdots & a_{2, j} \\ \vdots & \vdots & \ddots & \vdots \\ a_{i, 1} & a_{i, 2} & \cdots & a_{i, j}\end{array}\right), a_{i, j}=\Phi\left(\mathbf{x}_{i}-\mathbf{x}_{j}\right), i=1,2,3, \ldots, n, j=1,2,3, \ldots, n$

Here, $\mathbf{F}=\left[f\left(x_{1}\right), f\left(x_{2}\right), f\left(x_{3}\right), \ldots, f\left(x_{n}\right)\right]^{\mathrm{T}}$ is the value of the low-fidelity function at $\mathbf{X}=\left\{x_{1}, x_{2}, x_{3}, \ldots, x_{n}\right\}$. 


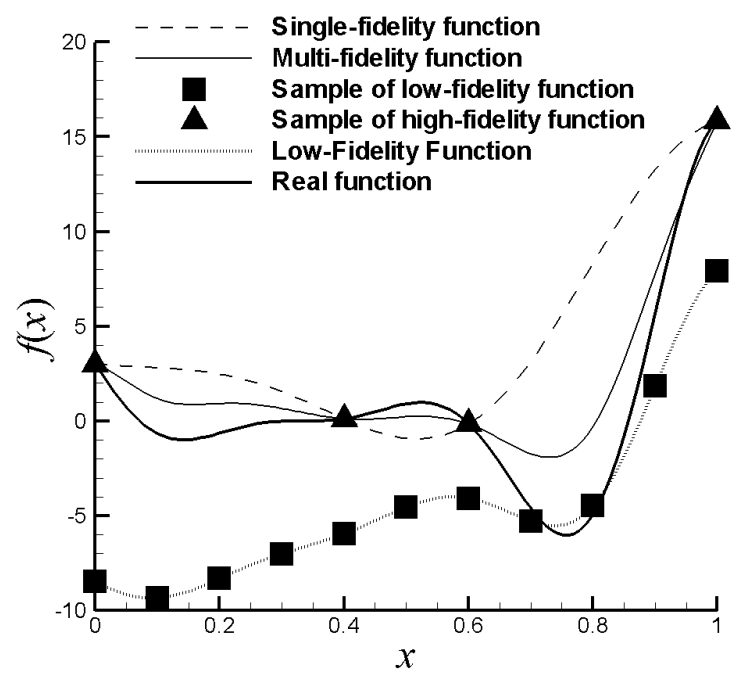

Figure 1. Schematic illustration of single-fidelity and multi-fidelity surrogate models.

\section{Efficient Global Optimization for the Multi-Objective Problem}

The procedure of EGO with the ordinary kriging model is illustrated in Figure 2a. The EGO process starts with the generated initial samples. In this study, the Latin hypercube sampling (LHS) is employed. Sample data are evaluated, and the model is predicted using the kriging method. An arbitrary optimization method can be used to find an additional point by maximizing an EHVI $[17,18]$. The EHVI is the function of the hypervolume improvement (HVI) combined with the uncertainty of the additional point. HVI is calculated from the hypervolume improvement of the additional sampling and the non-dominated solution shown in Figure 3a.

The EHVI at a point can be expressed as:

$$
\begin{aligned}
& \operatorname{EHVI}\left[f_{1}(\mathbf{x}), f_{2}(\mathbf{x}), \ldots, f_{M}(\mathbf{x})\right]= \\
& \int_{-\infty}^{f_{\text {ref } 1}} \int_{-\infty}^{f_{\text {ref } 2}} \ldots \int_{-\infty}^{f_{\text {refM }}} H V I\left[f_{1}(\mathbf{x}), f_{2}(\mathbf{x}), \ldots, f_{M}(\mathbf{x})\right] \times \phi_{1}\left(F_{1}\right) \phi_{2}\left(F_{2}\right) \ldots \phi_{M}\left(F_{M}\right) d F_{1} d F_{2} \ldots d F_{M},
\end{aligned}
$$

where $F_{i}$ denotes the Gaussian random variable $N\left[\hat{f}_{i}(\mathbf{x}), \hat{s}_{i}^{2}(\mathbf{x})\right] . \phi_{i}\left(F_{i}\right)$ is the probability density function and $f_{r e f i}$ is the reference value used for calculating the hypervolume. The maximization of EHVI is considered as the updating criterion to determine the location of an additional sample point. In this study, the hypervolume is calculated based on the HypEalgorithm [24], which is an algorithm that uses the Monte Carlo simulation [25] to approximate the hypervolume for multi/many-objective optimization problems. The Monte Carlo simulation is one of the simplest ways to calculate the hypervolume (HV) for many dimensions, which is often difficult to calculate. Thus, the HypE algorithm uses the benefit of the Monte Carlo simulation to calculate the hypervolume. The hypervolume is the volume of the non-dominated solutions measured from the reference point. The schematic illustration of the hypervolume is shown in Figure 3b. 


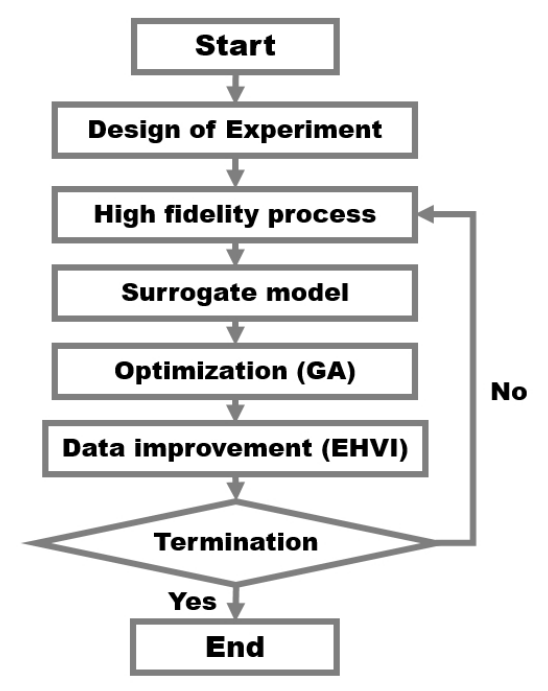

(a)

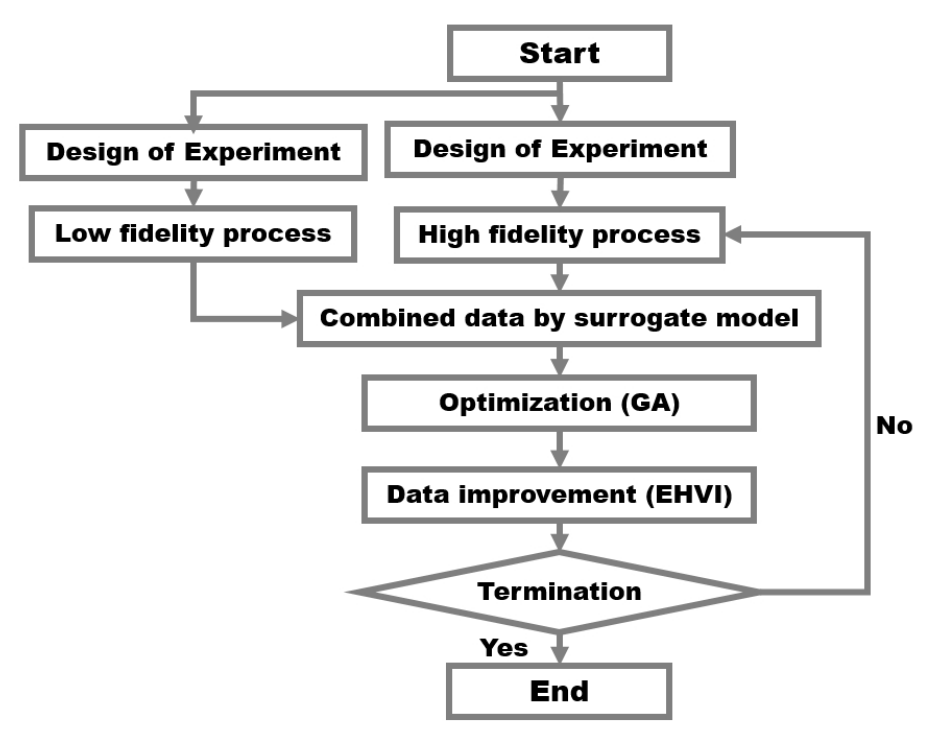

(b)

Figure 2. Flowchart of efficient global optimization: (a) single-fidelity efficient global optimization (EGO); (b) multi-fidelity EGO.

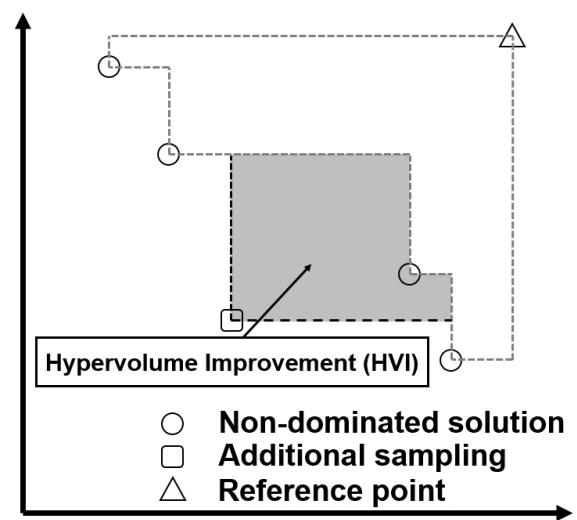

(a)

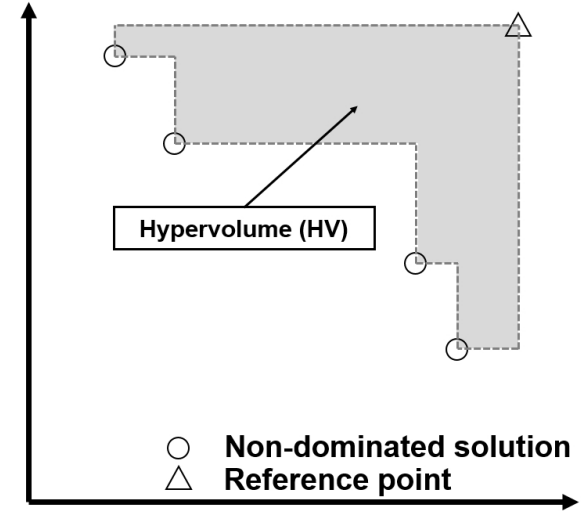

(b)

Figure 3. Schematic illustration of hypervolume improvement and hypervolume: (a) hypervolume improvement; (b) hypervolume.

The basic idea of the original EGO, namely EHVI-based explanation, can also be applied to the hybrid surrogate model expressed in (10), because local deviations are estimated using the kriging method. The procedure of EGO with the hybrid surrogate model proposed in this study is shown in Figure $2 \mathrm{~b}$. The proposed hybrid surrogate-model-based EGO starts by acquiring initial samples for a low-fidelity/low-cost function. The low-fidelity sample data are used to predict the global model; then, a set of samples for a high-fidelity function is obtained. This result is used to estimate the local deviations using the kriging method. Then, the multi-fidelity surrogate model, which can predict the unknown point value (an approximation of the high-fidelity function), is generated. A GA [23] is used to find the maximum EHVI point, $x$. In this study, the roulette wheel method was used in the selection process. Blend crossover (BLX)-0.5 [26] was used in the crossover process, and uniform mutation [27] with a mutation rate of 0.1 was used in the mutation process. 


\section{Investigation of Proposed Method by Solving Test Functions}

\subsection{Formulation}

The proposed multi-objective multi-fidelity EGO is investigated by solving two test functions; one has two objective functions, and the other has three objective functions. The results are compared with those of a kriging-based multi-objective EGO. The high-fidelity function is denoted by $f$, and the low-fidelity function is denoted by $f_{1}$.

The definition of two-objective optimization problem from Van Valedhuizen's test suite [28] is:

$$
\begin{aligned}
& \text { Minimize: } f_{1}\left(x_{1}, x_{2}, \ldots, x_{5}\right)=1-\exp \left(-\sum_{i=1}^{5}\left(x_{i}-1 / \sqrt{5}\right)^{2}\right) \\
& \text { Minimize: } f_{2}\left(x_{1}, x_{2}, \ldots, x_{5}\right)=1-\exp \left(-\sum_{i=1}^{5}\left(x_{i}+1 / \sqrt{5}\right)^{2}\right) \\
& f_{11}\left(x_{1}, x_{2}, \ldots, x_{5}\right)=1-\exp \left(-\sum_{i=1}^{5}\left(0.5 x_{i}-0.05-1 / \sqrt{5}\right)^{2}\right) \\
& f_{12}\left(x_{1}, x_{2}, \ldots, x_{5}\right)=1-\exp \left(-\sum_{i=1}^{5}\left(0.75 x_{i}+0.2+1 / \sqrt{5}\right)^{2}\right) .
\end{aligned}
$$

The design space of this problem is $x_{1}, x_{2}, \ldots, x_{5} \in[-2.5,2.5]$.

The definition of the DTLZ2 three-objective optimization problem [28] is:

$$
\begin{aligned}
& \text { Minimize: } f_{1}\left(x_{1}, x_{2}, \ldots, x_{5}\right)=(1+g) \cos \left(0.5 x_{i} \pi\right) \cos \left(0.5 x_{2} \pi\right) \\
& \text { Minimize: } f_{2}\left(x_{1}, x_{2}, \ldots, x_{5}\right)=(1+g) \cos \left(0.5 x_{1} \pi\right) \sin \left(0.5 x_{2} \pi\right) \\
& \text { Minimize: } f_{3}\left(x_{1}, x_{2}, \ldots, x_{5}\right)=(1+g) \sin \left(0.5 x_{1} \pi\right) \\
& g=\sum_{i=3}^{5}\left(x_{i}-0.5\right)^{2} \\
& f_{11}\left(x_{1}, x_{2}, \ldots, x_{5}\right)=\left(0.5+1.5 g_{c}\right) \cos \left(0.6 x_{i} \pi\right) \cos \left(0.4 x_{2} \pi\right) \\
& f_{12}\left(x_{1}, x_{2}, \ldots, x_{5}\right)=\left(0.3+1.2 g_{c}\right) \cos \left(0.4 x_{1} \pi\right) \sin \left(0.6 x_{2} \pi\right) \\
& f_{13}\left(x_{1}, x_{2}, \ldots, x_{5}\right)=\left(0.4+1.3 g_{c}\right) \sin \left(0.5 x_{1} \pi\right) \\
& g_{c}=\sum_{i=3}^{5}\left(0.8 x_{i}-0.3\right)^{2} .
\end{aligned}
$$

The design space of this problem is $x_{1}, x_{2}, \ldots, x_{5} \in[0,1]$.

In each investigation, 10 initial high-fidelity points, $f$, and 150 low-fidelity points, $f_{1}$, were acquired by LHS. The number of iterations was set to 30 for each test function.

\subsection{Two-Objective Test Function Results}

The solution space acquired by the proposed multi-fidelity/multi-objective EGO is compared with that acquired by the single-fidelity/multi-objective EGO as shown in Figure 4. Because the single-fidelity/multi-objective EGO finds local optimum points at the beginning of the optimization process, the solution for additional samples stalls earlier. On the other hand, the developed multi-fidelity/multi-objective EGO can find a solution close to the global optimum solution of this multi-objective optimization problem. The histories of the hypervolumes of the two methods are compared in Figure 5. These results also indicate that the proposed multi-fidelity/multi-objective EGO provides better solutions, which shows higher diversity because its hypervolume is larger than that of the single-fidelity/multi-objective EGO. These results also suggest that the proposed multi-fidelity/multi-objective EGO obtains better solutions than the single-fidelity/multi-objective 
EGO, because the non-dominated solutions of the former can dominate all the non-dominated solutions of the latter. In addition, comparisons of the hypervolumes show that the solution of additional samples by the single-fidelity/multi-objective EGO continues to stall earlier.

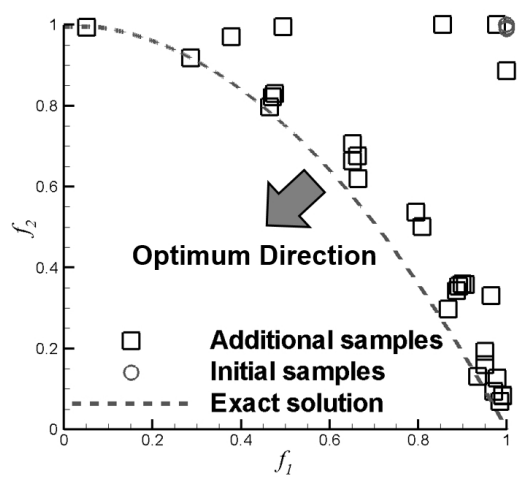

(a)

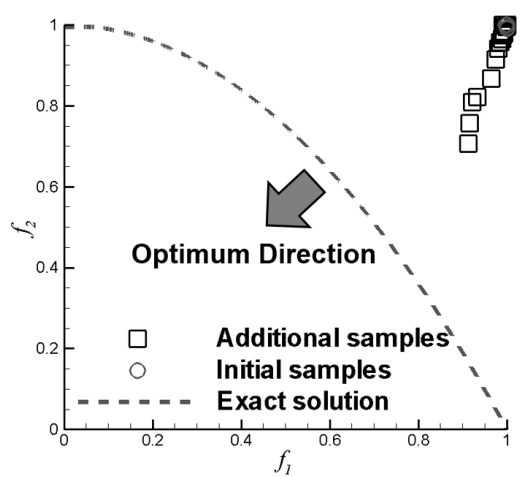

(b)

Figure 4. Initial sampling data and additional sampling data of two-objective test problem: (a) multi-fidelity approach; (b) single-fidelity approach.

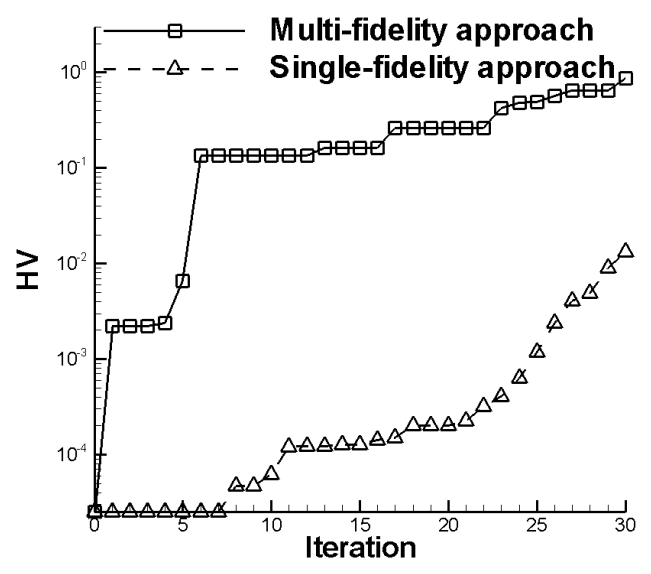

Figure 5. Hypervolume comparison of multi-fidelity approach and single-fidelity approach of two-objective test problem.

To investigate the reason for the superiority of the proposed multi-fidelity/multi-objective EGO, the cross-validation $[29,30]$ of $f_{1}$ and $f_{2}$ was compared, as shown in Figure 6. It can be seen that the linear regression line nearly coincides with the predicted line in the case of the proposed multi-fidelity/multi-objective EGO. Thus, the multi-fidelity surrogate model achieves higher accuracy than the single-fidelity surrogate model. More specifically, the proposed multi-fidelity/multi-objective EGO achieves higher accuracy because the dataset obtained by low-fidelity evaluation enables the surrogate model to predict the solution in the uncertainty region. 
The developed approach

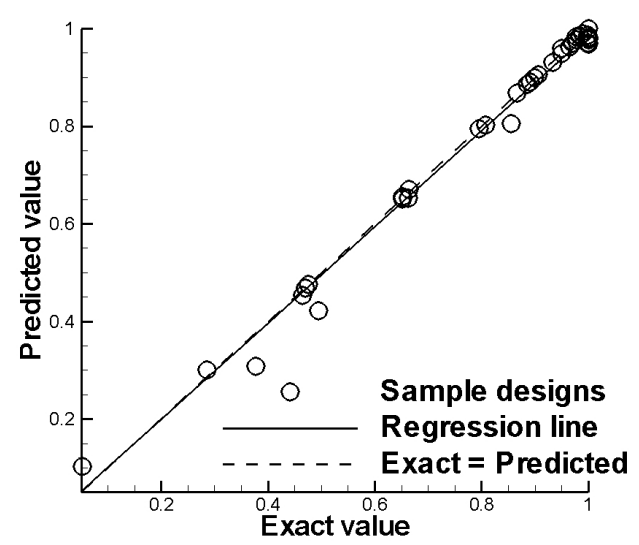

The developed approach

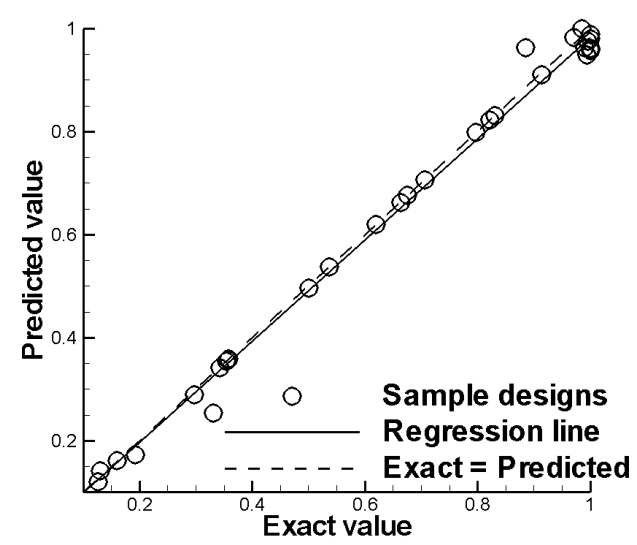

The single-fidelity

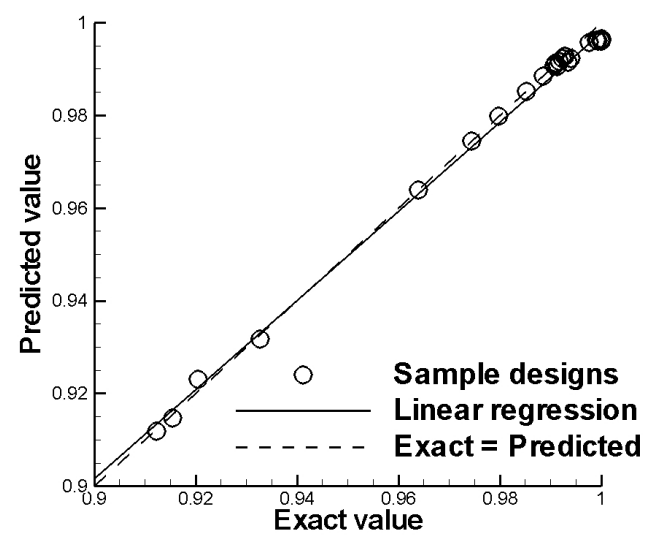

(a)

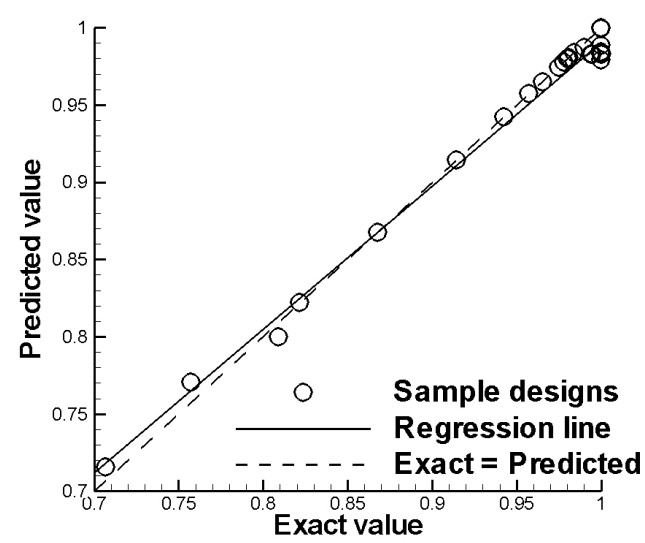

(b)

Figure 6. Cross-validation results of the two-objective test problem: (a) result of $f_{1}$; (b) result of $f_{2}$.

\subsection{Three-Objective Test Function Results}

Further, all the samples acquired by the two methods (the proposed multi-fidelity/multi-objective EGO and the single-fidelity/multi-objective EGO) are compared as shown in Figure 7. According to these results, the proposed multi-fidelity/multi-objective EGO and the single-fidelity/multi-objective EGO have similar non-dominated solutions. However, the results of the multi-fidelity/multi-objective EGO are better than those of the single-fidelity/multi-objective EGO because some of the results of additional sampling by the single-fidelity/multi-objective EGO stall at local optimum points. The hypervolumes of the two methods are compared in Figure 8. The hypervolume comparison of the proposed multi-fidelity/multi-objective EGO and single-fidelity/multi-objective EGO is shown in Figure 9. It suggests that the proposed multi-fidelity/multi-objective EGO gives better solutions than that of the single-fidelity/multi-objective EGO. These results indicate that the proposed multi-fidelity/multi-objective EGO has a higher convergence rate than the single-fidelity/multi-objective EGO. The solution of the proposed multi-fidelity/multi-objective EGO converges after 18 iterations with a larger hypervolume, whereas the solution of the single-fidelity/multi-objective EGO converges after 23 iterations. 


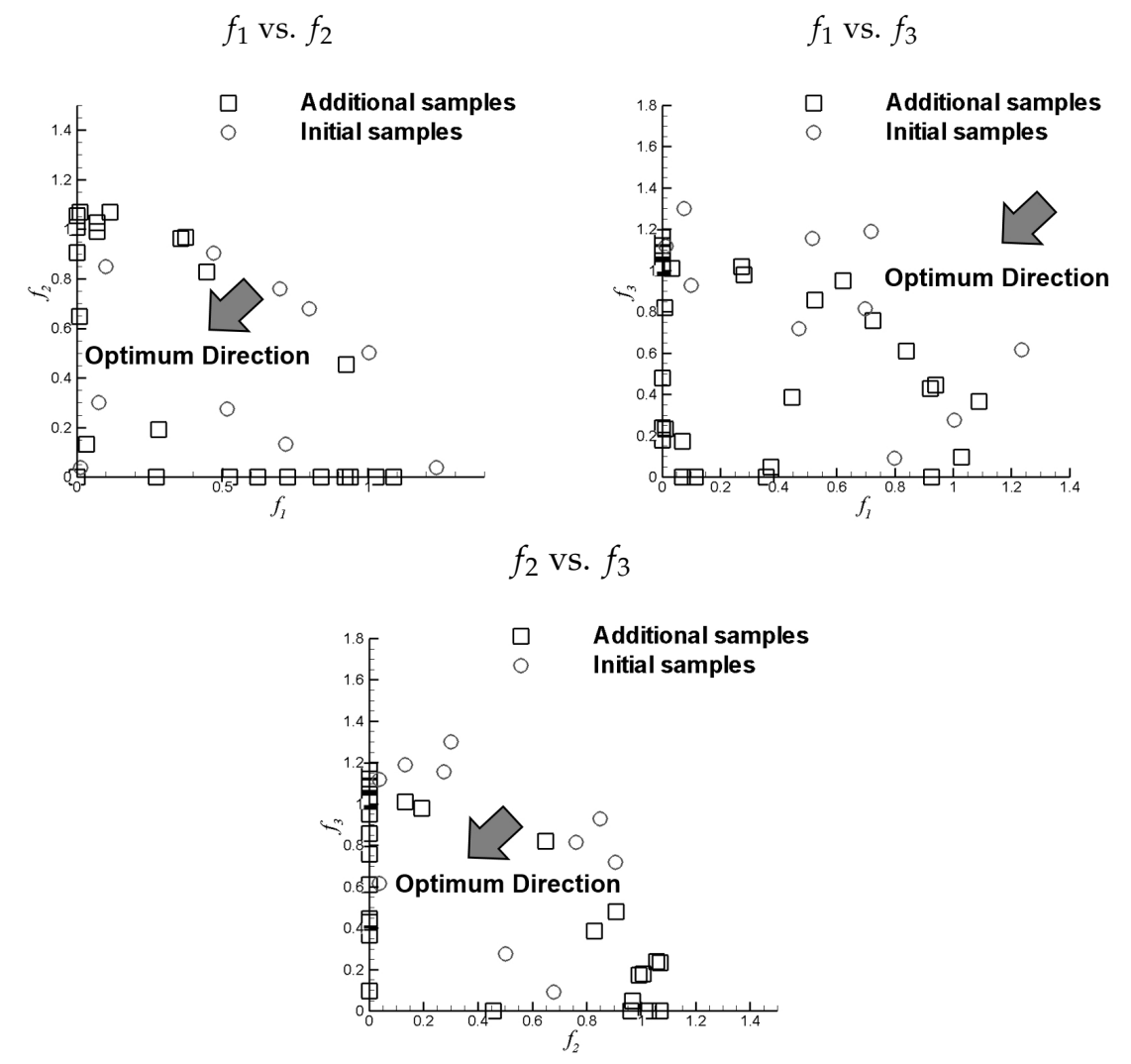

(a)
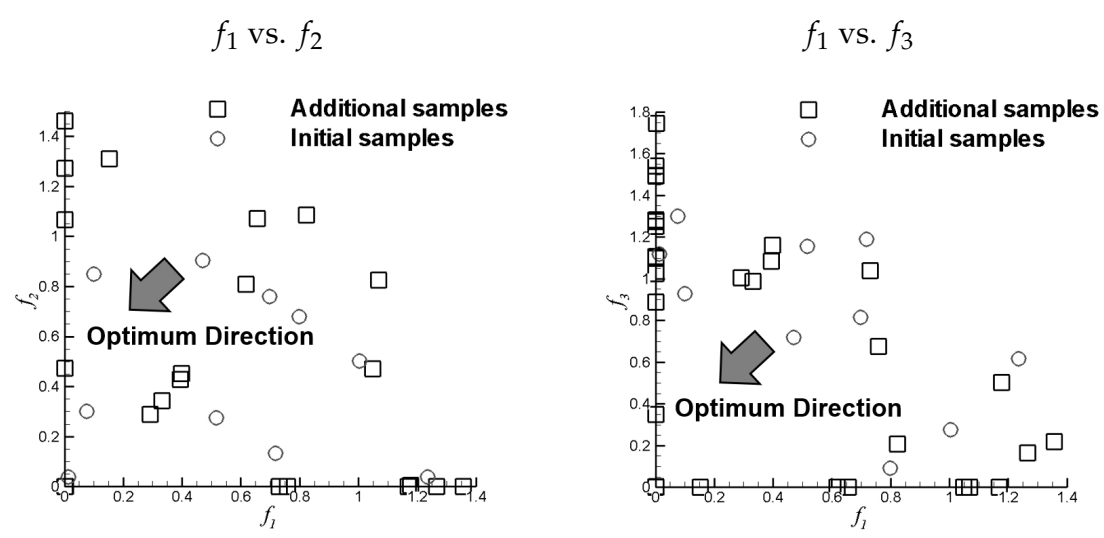

$f_{2}$ vs. $f_{3}$

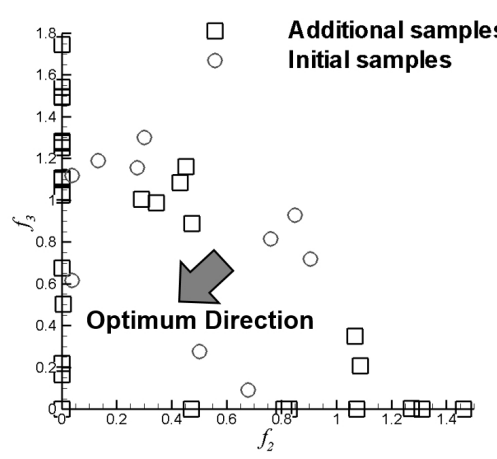

(b)

Figure 7. Initial sampling data and additional sampling data of three-objective test problem: (a) multi-fidelity approach; (b) single-fidelity approach. 


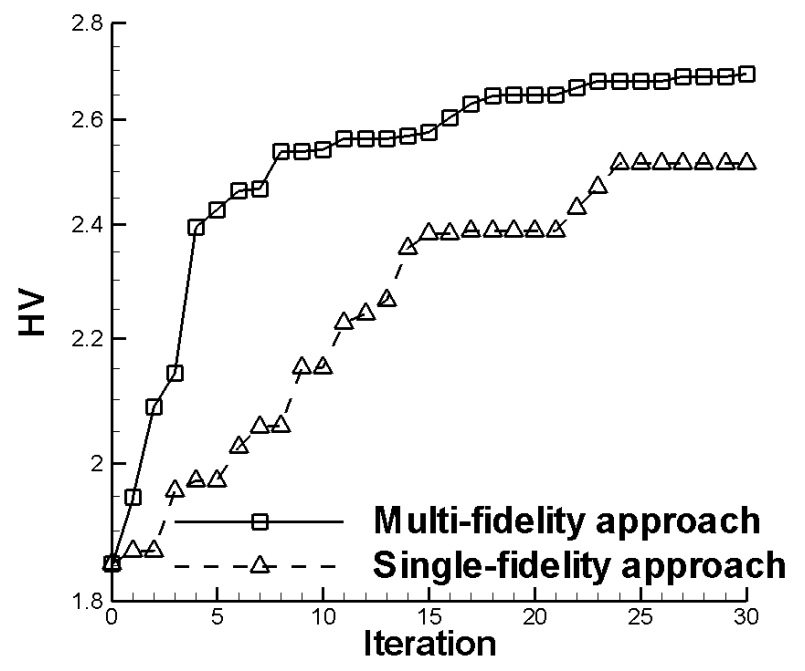

Figure 8. Hypervolume comparison of the multi-fidelity approach and the single-fidelity approach of three-objective test problem.

The developed approach

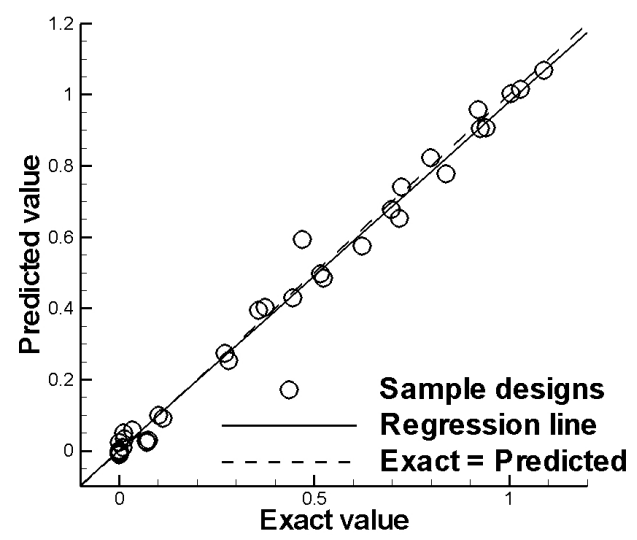

The developed approach

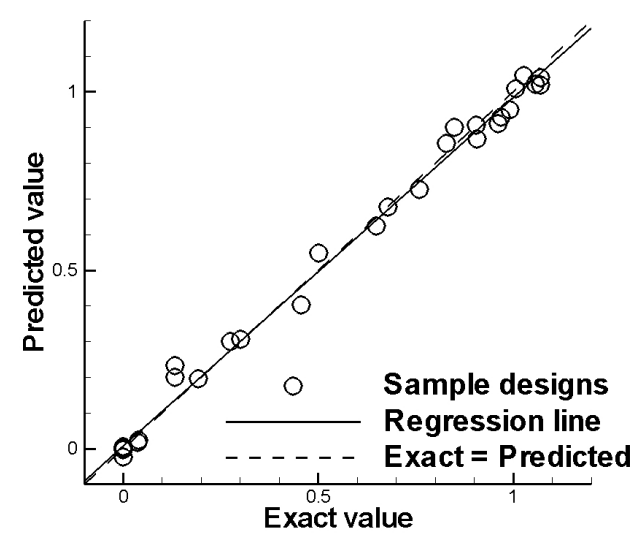

The single-fidelity

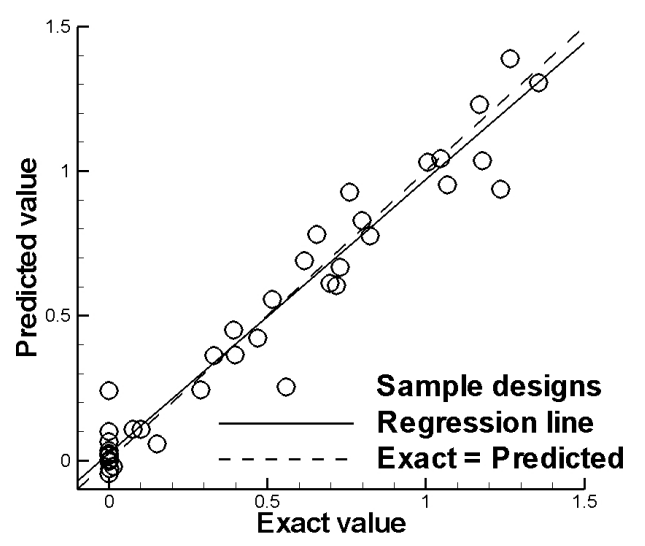

(a)

The single-fidelity

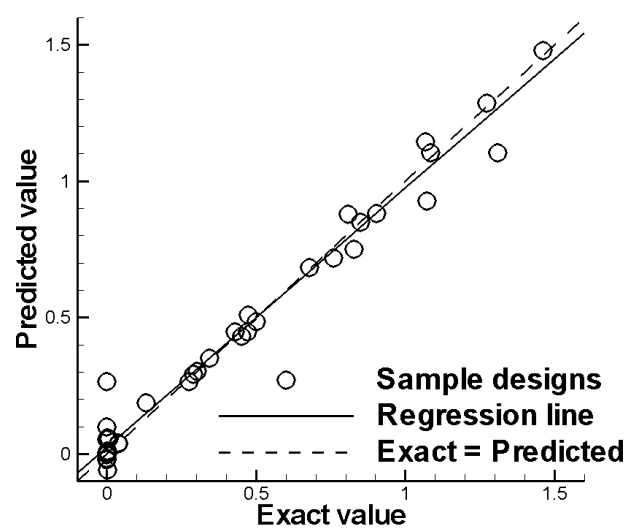

(b)

Figure 9. Cont. 
The developed approach

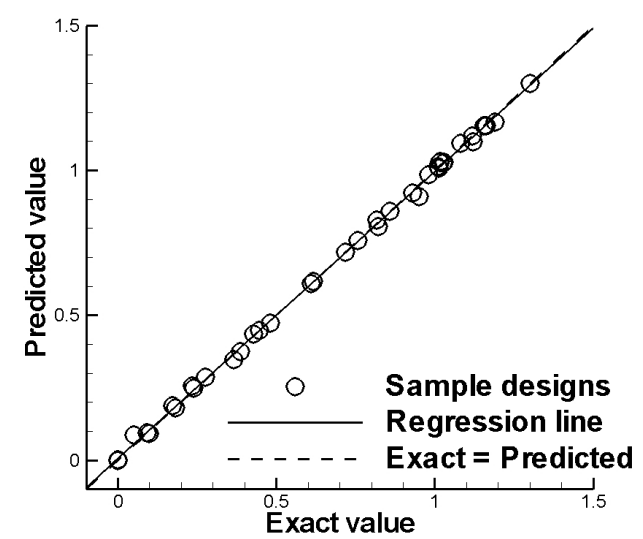

The single-fidelity

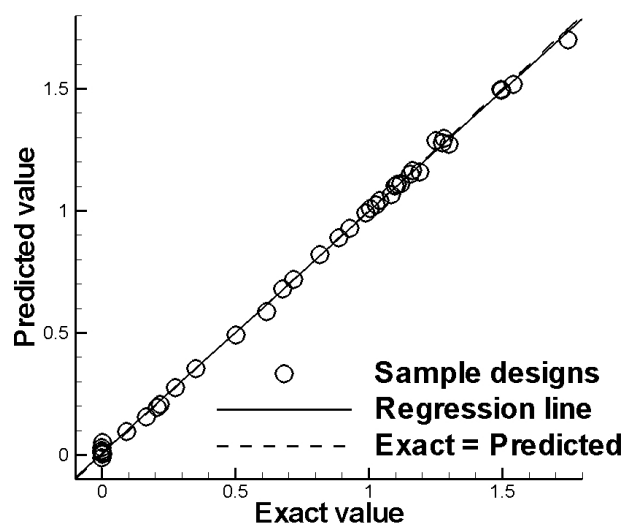

(c)

Figure 9. Cross-validation results of the three-objective test problem: (a) result of $f_{1} ;(\mathbf{b})$ result of $f_{2}$; (c) result of $f_{3}$.

The cross-validation results for $f_{1}, f_{2}$ and $f_{3}$ are shown in Figure 9. The results indicate that the multi-fidelity surrogate model can achieve higher accuracy than the single-fidelity surrogate model for $f_{1}$ and $f_{2}$. Moreover, the results show that the non-dominated solution of the multi-fidelity/multi-objective EGO has a higher convergence rate because of the higher accuracy of the multi-fidelity surrogate model. With regard to $f_{3}$, a comparison between the multi-fidelity and single-fidelity surrogate models shows that the two methods have similar accuracy because $f_{3}$ has a simpler shape than $f_{1}$ and $f_{2}$.

\section{Airfoil Design Problem}

The proposed multi-fidelity/multi-objective design method discussed in Section 3 was also investigated by solving two airfoil design problems as real-world design problems.

\subsection{Formulations}

\subsubsection{Two-Objective Case}

The first problem has two objectives: minimize the aerodynamic drag $\left(C_{\mathrm{d}}\right)$ at Mach 0.3 , which requires a target lift of 0.5 , and maximize the airfoil thickness at $75.0 \%$ of the chord length $\left(t_{75}\right)$, which can be obtained directly by the real function because it can be calculated rapidly. Thus, two surrogate models are constructed. The optimization problem can be expressed as:

$$
\begin{aligned}
& \text { Minimize: } C_{\mathrm{d}} \text { at } R e=4 \times 10^{6}, M a=0.3, C_{1}=0.5 \\
& \text { Maximize: } t_{75}
\end{aligned}
$$

The number of initial samples for the high-fidelity/high-cost function is set to 10, and the number of low-fidelity/low-cost functions for the multi-fidelity surrogate model is 150. Further, the number of additional samples for this problem is set to $30 . t_{75}$ can be immediately calculated after the set of design parameters is decided upon. Thus, we used the exact value for $t_{75}$ in the following equation based on Equation (16).

$$
E H V I=\int_{-\infty}^{C_{\mathrm{d}, \max }} H V I\left[C_{\mathrm{d}}, t_{75}\right] \times \phi_{1}\left(C_{\mathrm{d}}\right) d C_{\mathrm{d}}
$$




\subsubsection{Three-Objective Case}

The second problem has three objectives: minimize $C_{\mathrm{d}}$ at cruising speed, maximize $t_{75}$ and maximize the lift coefficient $\left(C_{1}\right)$ in the landing condition at an angle of attack of $5.0^{\circ}$. The optimization problem is expressed as:

$$
\begin{aligned}
& \text { Minimize: } C_{\mathrm{d}} \text { at } R e=4 \times 10^{6}, M a=0.3, C_{1}=0.5 \\
& \text { Maximize: } C_{1} \text { at } R e=2 \times 10^{6}, M a=0.15, \alpha=5.0^{\circ} \\
& \text { Maximize: } t_{75}
\end{aligned}
$$

In this problem, the hybrid surrogate model is used to predict $C_{\mathrm{d}}$ and $C_{1}$. Further, $t_{75}$ can be directly obtained by the real function. The number of initial samples for the high-fidelity/high-cost function is set to 10 , and the number of low-fidelity/low-cost functions for the multi-fidelity surrogate model is 150. Further, the number of additional samples for this problem is set to $30 . t_{75}$ can be immediately calculated after the set of design parameters is decided upon. Thus, we used the exact value for $t_{75}$ in the following equation based on Equation (16).

$$
E H V I=\int_{-\infty}^{C_{\mathrm{d}, \max }} \int_{C_{1, \text { min }}}^{\infty} H V I\left[C_{\mathrm{d}}, t_{75}, C_{1}\right] \times \phi_{1}\left(C_{\mathrm{d}}\right) \phi_{2}\left(C_{1}\right) d C_{\mathrm{d}} d C_{1} .
$$

\subsection{Evaluation Methods}

\subsubsection{High-Fidelity Evaluation Using CFD as the High-Cost Function}

The aerodynamic evaluation as a high-fidelity function was carried out using a Reynolds-averaged Navier-Stokes (RANS) solver [31]. The governing equation is expressed as:

$$
\frac{\partial}{\partial T} \int_{\Omega} \Psi d V+\oint_{\partial \Omega} Q \cdot n d s=0
$$

$\Psi$ is a vector that consists of conservative quantities, and $Q$ is the summation of conservative quantities entering and leaving the area. A lower-upper symmetric Gauss-Seidel (LU-SGS) implicit method [32] was employed for time integration, and a third-order-accurate upwind difference scheme with a monotone upstream-centered scheme for conservation laws (MUSCL) method [33] was employed for the flux evaluation. Further, the Baldwin-Lomax model [34] was used as a turbulent model. In addition, a structured grid was automatically created by an algebraic method for each design ( $200 \times 61$ structured grid for the RANS solver, as shown in Figure 10). The computation time of CFD in this work is approximately 180 to $300 \mathrm{~s}$.

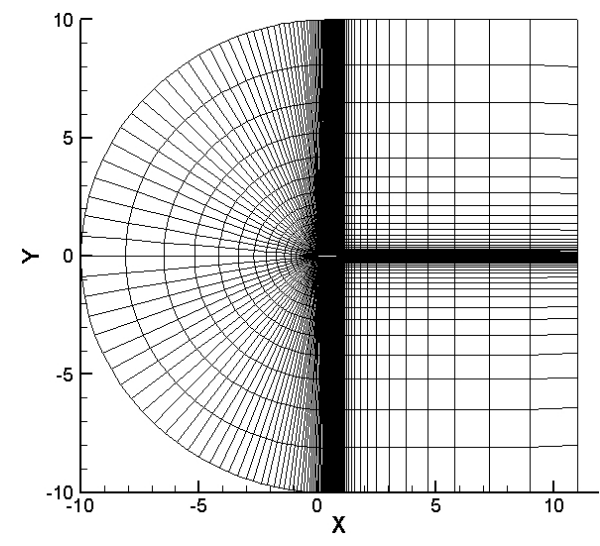

(a)

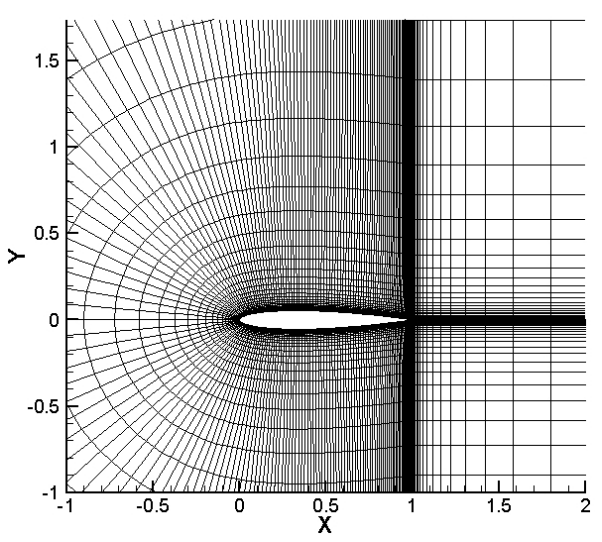

(b)

Figure 10. Computation structured grid: (a) full length; (b) grid around the airfoil. 


\subsubsection{Low-Fidelity Solver as the Low-Cost Function}

XFOIL [35] was employed as the low-fidelity evaluation. In XFOIL, the inviscid pressure distribution is modeled using a linear vortex strength distribution, while the viscous effects and the development of the laminar-turbulent boundary layer are modeled using integral boundary layer theory. The computation time of XFOIL is approximately 1 to $2 \mathrm{~s}$.

\subsubsection{Airfoil Representation}

In this study, the class-shape function transformation (CST) parameterization method [36] was used for airfoil shape parameterization. The benefits of CST are that it could can generate several kinds of aerodynamic shapes and that it has a high degree of freedom due to the changeable number of numbers of the controlled parameter to generate the airfoil shape; whereas the other type of airfoil representation, such as NACA's airfoil representation, parametric section (PARSEC) and non-uniform rational B-spline (NURBS), have the fixed controlled parameters. The product of a class function, $C(x / c)$, and a shape function, $S(x / c)$, can be represented geometrically by adding a term that characterizes the trailing edge thickness:

$$
\frac{y}{c}=C\left(\frac{x}{c}\right) S\left(\frac{x}{c}\right)+\frac{x}{c} \frac{\Delta z_{t e}}{c},
$$

where $\Delta z_{t e}$ is the trailing edge thickness, and $C(x / c)$ is given in generic form by:

$$
C\left(\frac{x}{c}\right) \equiv\left(\frac{x}{c}\right)^{N_{1}}\left[1-\frac{x}{c}\right]^{N_{2}} \text { for } 0 \leq \frac{x}{c} \leq 1 .
$$

The shape function, $S(x / c)$, is defined on the basis of the Bernstein binomials [37], by the introduction of weight factor $b_{i}$ as follows:

$$
S\left(\frac{x}{c}\right)=\sum_{i=0}^{n}\left[b_{i} \cdot K_{i, p} \cdot\left(\frac{x}{c}\right)^{i} \cdot\left(1-\frac{x}{c}\right)^{p-i}\right],
$$

where $p$ is the degree of the Bernstein binomials. In this study, $N_{1}$ was set to 0.5 and $N_{2}$ was set to 1.0 . Further, third-degree Bernstein polynomials were used to generate the airfoil shape for the lower side $b_{1}-b_{3}$ and the upper side $b_{4}-b_{6}$. The ranges of the design parameters are defined in Table 1.

Table 1. The range of design variables for airfoil design by class-shape function transformation (CST).

\begin{tabular}{cc}
\hline Design Parameter & Design Range \\
\hline$b_{1}$ & $-0.18--0.01$ \\
$b_{2}$ & $-0.15--0.05$ \\
$b_{3}$ & $-0.18--0.02$ \\
$b_{4}$ & $0.10-0.18$ \\
$b_{5}$ & $0.05-0.15$ \\
$b_{6}$ & $0.05-0.15$ \\
\hline
\end{tabular}

\subsection{Results}

\subsubsection{Two-Objective Airfoil Shape Optimization Results}

All the samples obtained by the two methods (the proposed multi-fidelity/multi-objective EGO and the single-fidelity/multi-objective EGO) are shown in Figure 11. These results show that the proposed multi-fidelity/multi-objective EGO can achieve greater diversity in the solution space than the single-fidelity/multi-objective EGO. The hypervolume of the proposed multi-fidelity/multi-objective EGO showed faster convergence (after 20 iterations) than that of 
the single-fidelity/multi-objective EGO (after 26 iterations). In addition, the non-dominated solutions of the proposed multi-fidelity/multi-objective EGO included $C_{\mathrm{d}}$ ranging from 0.006 to 0.027 and $t_{75}$ ranging from 0.034 to 0.064 . On the other hand, the non-dominated solutions of the single-fidelity /multi-objective EGO included $C_{\mathrm{d}}$ ranging from 0.009 to 0.016 and $t_{75}$ ranging from 0.047 to 0.064 . The histories of the hypervolumes of the two methods are compared in Figure 12. According to these results, the single-fidelity surrogate model could find only local optimum points, whereas the proposed multi-fidelity approach could find global optimum points.

The cross-validation results for $C_{\mathrm{d}}$ are shown in Figure 13. It can be confirmed that the hybrid surrogate model achieves higher accuracy than the single-fidelity kriging-based surrogate model. This is because the low-fidelity data enable the surrogate model to predict the data in the uncertainty region. Thus, the multi-fidelity/multi-objective EGO achieves faster solution converge because the multi-fidelity surrogate model achieves higher accuracy than the single-fidelity surrogate model. Because the proposed multi-fidelity surrogate model achieves higher accuracy, the optimization process based on it is more likely to obtain global optimum points.

The optimal shapes of the non-dominated solutions of the two methods that minimize $C_{d}$ and maximize $t_{75}$ are compared in Figure 14. The optimal designs of the single-fidelity/multi-objective EGO have similar shapes because the algorithm converges early at these optimum points. On the other hand, the optimal designs of the proposed multi-fidelity/multi-objective EGO have different shapes for each objective. In addition, the total design time of the multi-fidelity/multi-objective EGO is $162 \mathrm{~min}$, and the total design time of the single-fidelity/multi-objective EGO is $160 \mathrm{~min}$.

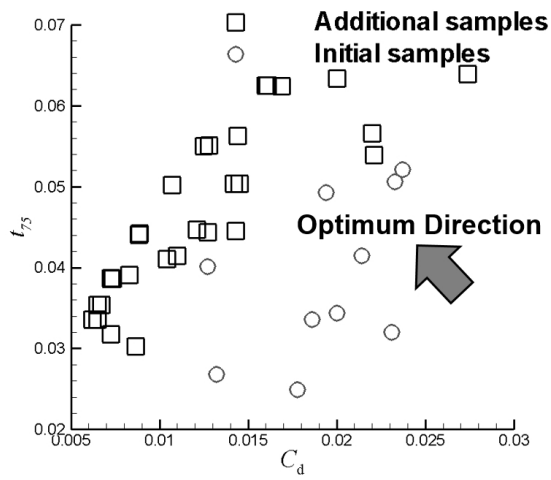

(a)

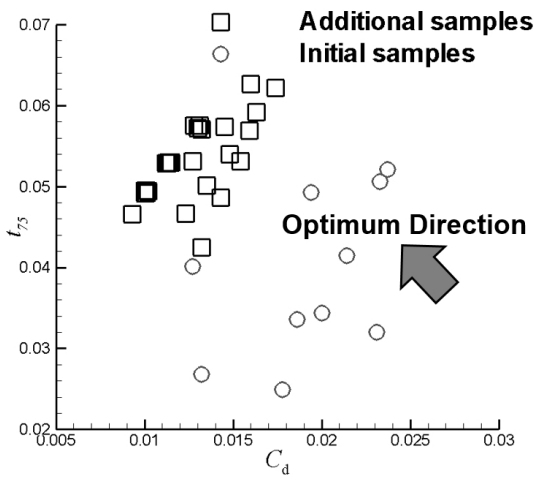

(b)

Figure 11. Initial sampling data and additional sampling data of two-objective airfoil shape optimization problem: (a) multi-fidelity approach; (b) single-fidelity approach.

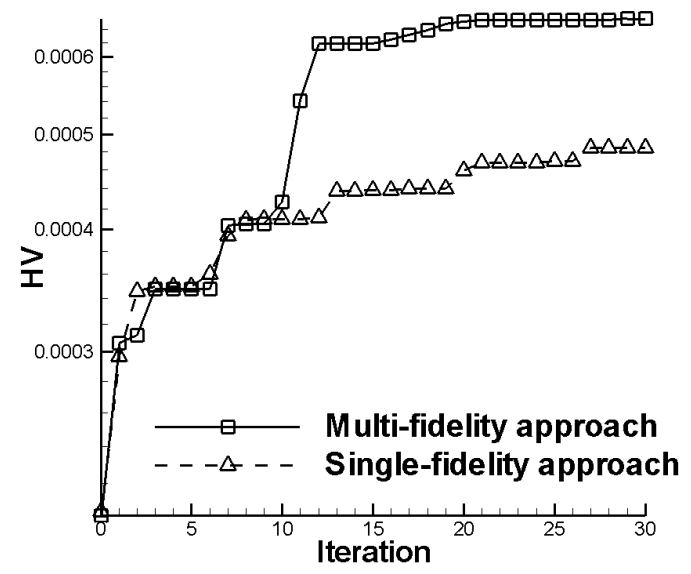

Figure 12. Hypervolume comparison of multi-fidelity approach and single-fidelity approach of two-objective airfoil shape optimization problem. 
The developed approach

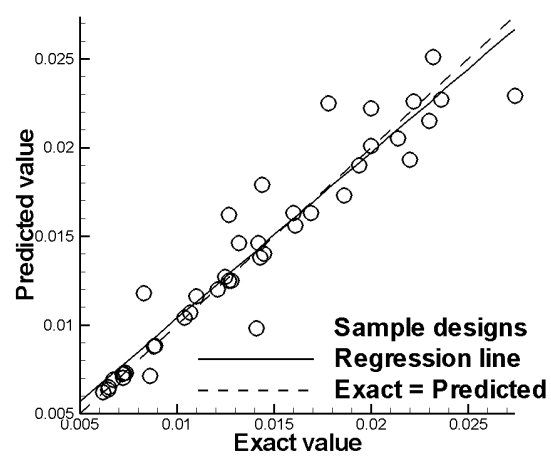

The single-fidelity

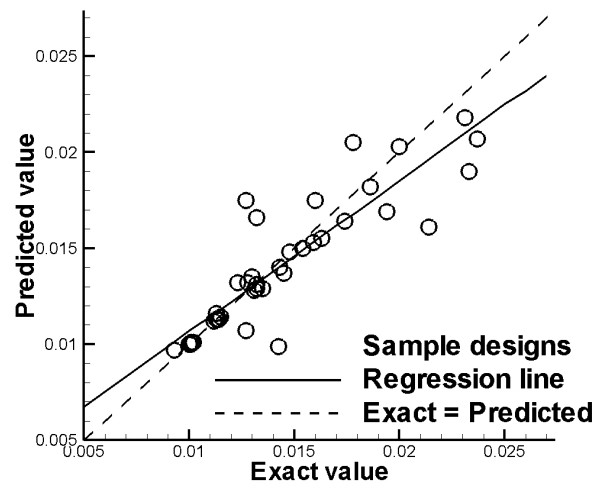

Figure 13. Cross-validation of two-objective airfoil shape optimization problem of $C_{\mathrm{d}}$.

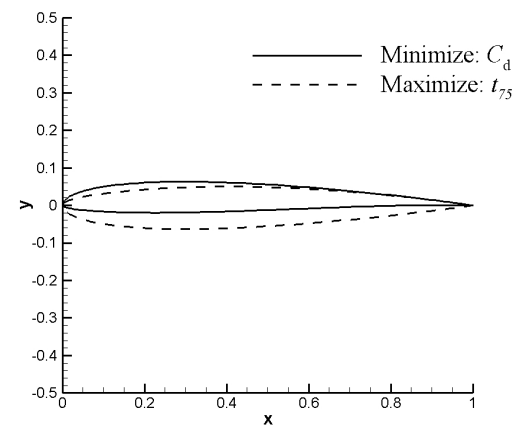

(a)

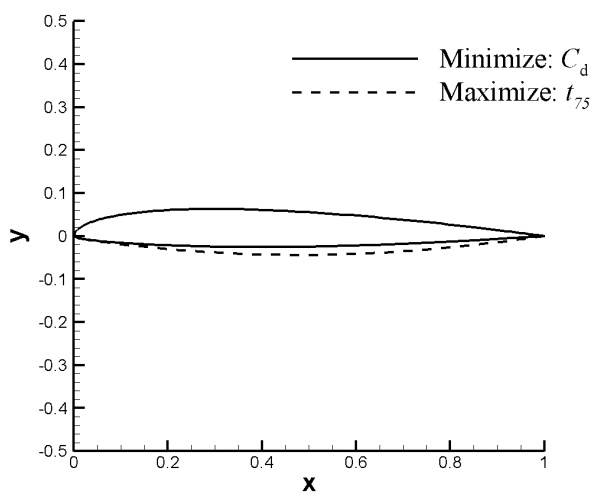

(b)

Figure 14. Comparison of design geometries of two-objective airfoil shape optimization problem: (a) multi-fidelity approach; (b) single-fidelity approach.

\subsubsection{Three-Objective Airfoil Shape Optimization Results}

All the samples obtained by the two methods (the proposed multi-fidelity/multi-objective EGO and the single-fidelity/multi-objective EGO) are compared in Figure 15. In addition, the histories of the hypervolumes of the two methods are compared in Figure 16. These results show that the proposed multi-fidelity/multi-objective EGO achieves greater diversity of the non-dominated solutions because its hypervolume is larger than that of the single-fidelity/multi-objective EGO. The non-dominated solutions of the proposed multi-fidelity/multi-objective EGO included $C_{\mathrm{d}}$ ranging from 0.007 to $0.022, t_{75}$ ranging from 0.034 to 0.065 and $C_{1}$ ranging from 0.0938 to 1.054 . On the other hand, the non-dominated solutions of the single-fidelity/multi-objective EGO included $C_{\mathrm{d}}$ ranging from 0.010 to $0.022, t_{75}$ ranging from 0.047 to 0.065 and $C_{1}$ ranging from 0.0938 to 1.039 . Thus, the proposed multi-fidelity/multi-objective EGO achieved greater diversity of the solutions than the single-fidelity/multi-objective EGO. The cross-validation results for $C_{\mathrm{d}}$ and $C_{1}$ are shown in Figure 17. It can be seen that the proposed hybrid surrogate model achieves higher accuracy than the single-fidelity kriging-based surrogate model. Thus, the proposed multi-fidelity/multi-objective EGO can achieve greater diversity of the solutions because its surrogate model achieves higher accuracy.

The optimal shapes of the non-dominated solutions of the two methods that minimize $C_{d}$, maximize $t_{75}$ and maximize $C_{1}$ are compared in Figure 18. The optimal designs of the single-fidelity/multi-objective EGO have similar shapes for minimizing $C_{\mathrm{d}}$ and maximizing $C_{1}$ because the algorithm converges early at these optimum points. On the other hand, the optimal designs of the proposed multi-fidelity/multi-objective EGO have different shapes for each objective. In addition, the total 
design time of the multi-fidelity/multi-objective EGO is $162 \mathrm{~min}$, and the total design time of the single-fidelity/multi-objective EGO is $160 \mathrm{~min}$.

$$
C_{\mathrm{d}} \text { vs. } C_{1}
$$

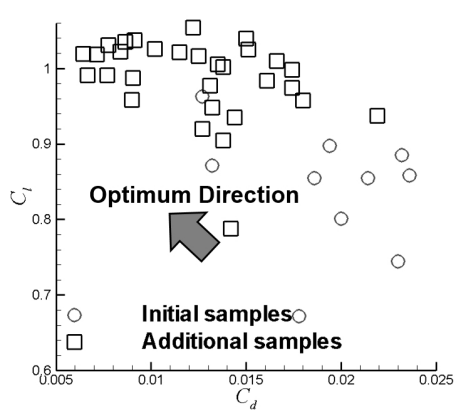

$C_{1}$ vs. $t_{75}$

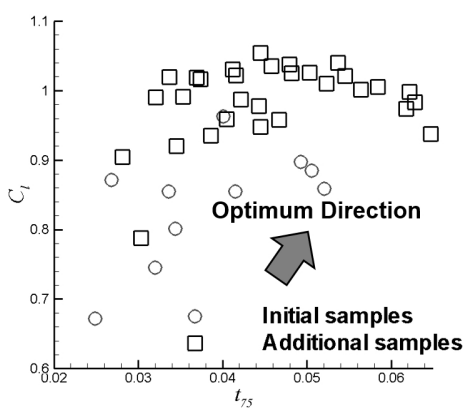

(a)

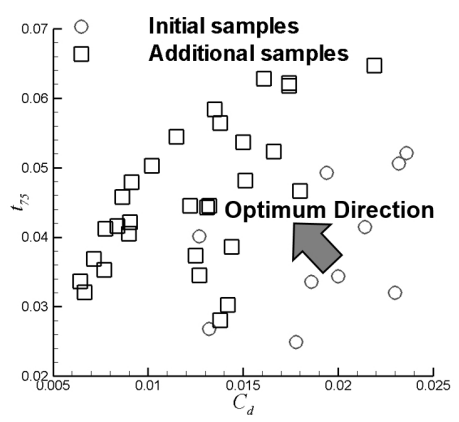

$C_{\mathrm{d}}$ vs. $t_{75}$

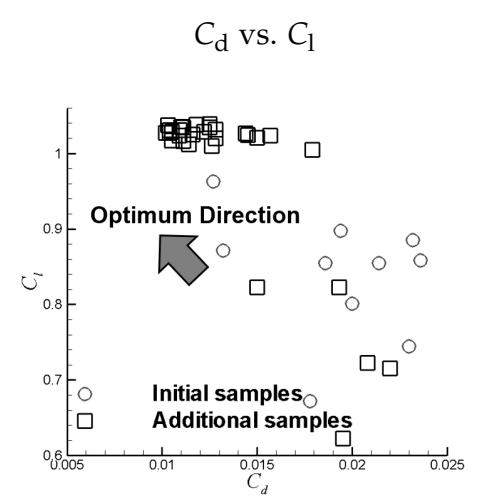

$C_{\mathrm{d}}$ vs. $t_{75}$

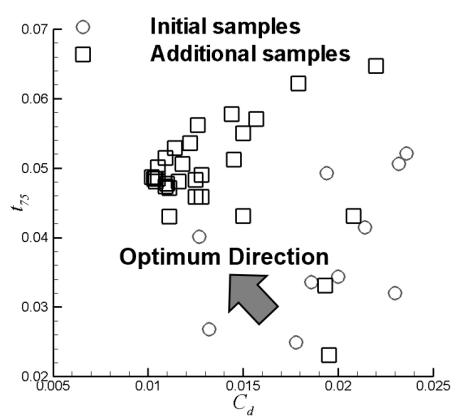

$C_{1}$ vs. $t_{75}$

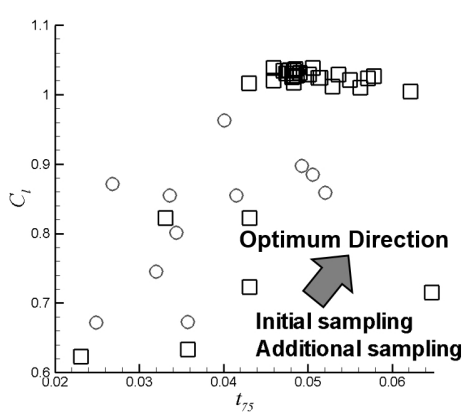

(b)

Figure 15. Initial sampling data and additional sampling data of three-objective airfoil shape optimization problem: (a) multi-fidelity approach; (b) single-fidelity approach. 


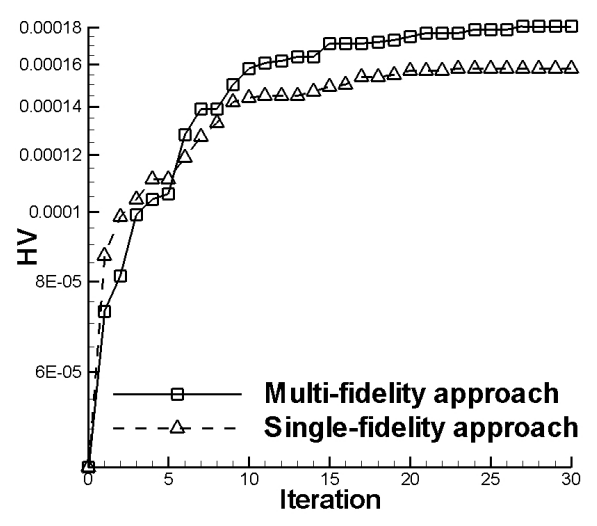

Figure 16. Hypervolume comparison of multi-fidelity approach and single-fidelity approach.

The developed approach

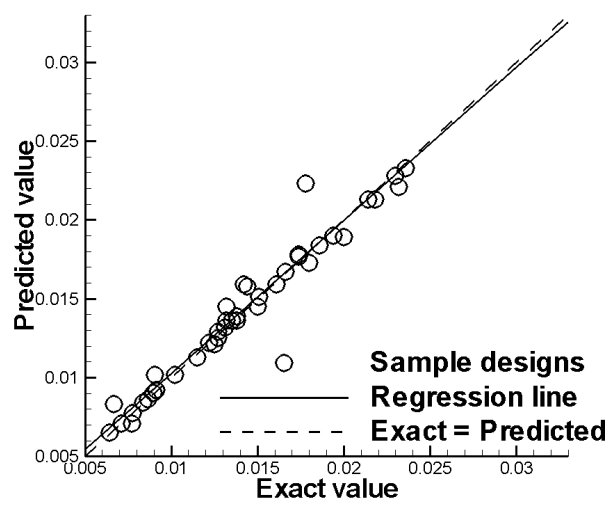

The developed approach

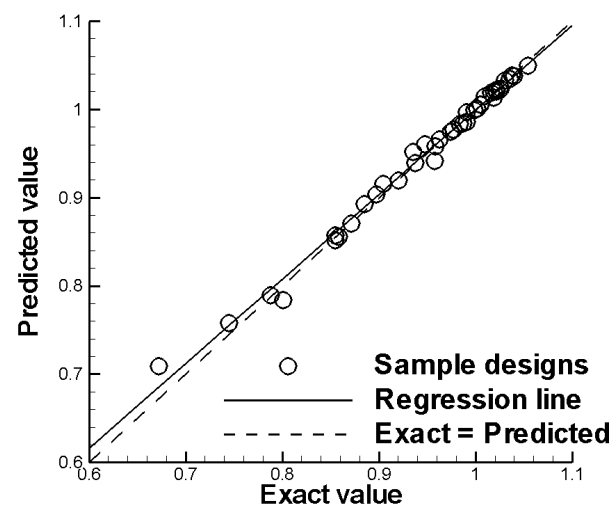

The single-fidelity

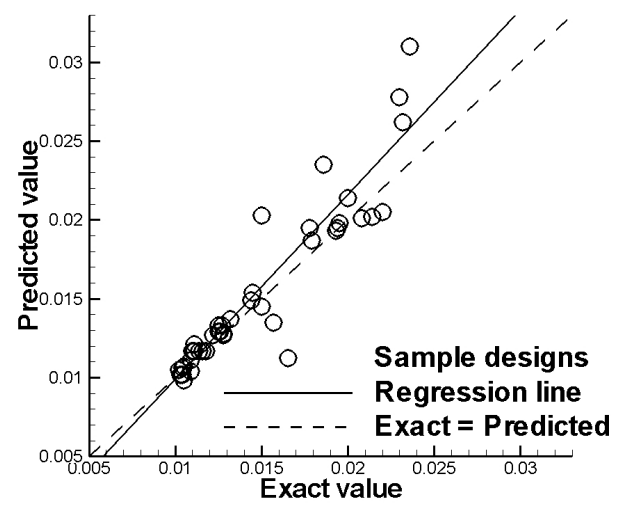

(a)

The single-fidelity

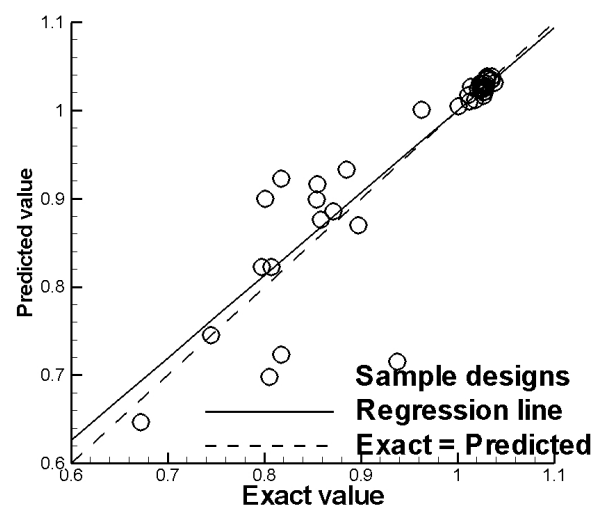

(b)

Figure 17. Cross-validation results of three-objective airfoil shape optimization problem: (a) results of $C_{\mathrm{d}} ;(\mathbf{b})$ results of $C_{1}$. 


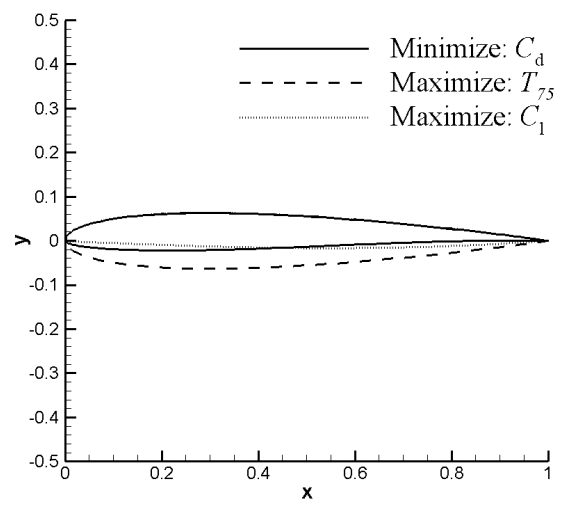

(a)

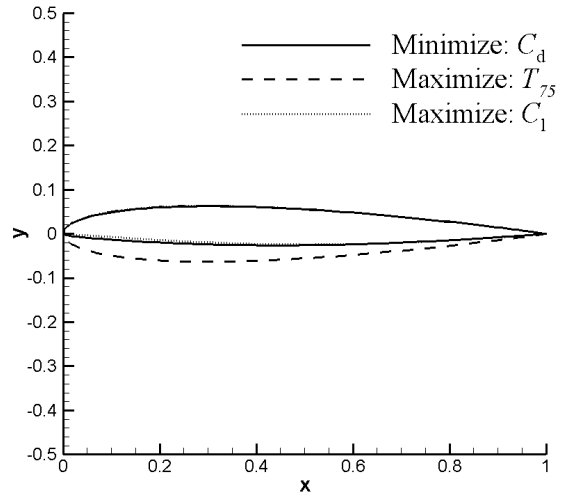

(b)

Figure 18. Comparison of design geometries of three-objective airfoil shape optimization problem: (a) multi-fidelity approach; (b) single-fidelity approach.

\section{Conclusions}

In this article, a multi-fidelity/multi-objective EGO combined with kriging and RBF was proposed based on the hybrid surrogate model to solve the multi-objective optimization problems and was applied to solve the multi-objective airfoil design problem. The proposed method constructed a hybrid surrogate model based on an RBF that predicts a global model and an ordinary kriging method that predicts the local variance. EHVI was used as an index to find additional samples for the optimization process. For multi-fidelity optimization, the global model was constructed using a dataset evaluated by a low-fidelity function, and the local variance was estimated using a dataset evaluated by a high-fidelity function.

To examine the proposed multi-fidelity/multi-objective EGO, two-/three-objective test problems were solved, and the results of the proposed multi-fidelity/multi-objective EGO were compared with those of the single-fidelity/multi-objective EGO. The results for the test functions showed that the proposed multi-fidelity/multi-objective EGO achieves faster convergence than the single-fidelity/multi-objective EGO. Moreover, the results showed that the proposed multi-fidelity/multi-objective EGO has greater diversity of the non-dominated solutions than the single-fidelity/multi-objective EGO. In addition, the results showed that the proposed multi-fidelity/multi-objective EGO has fewer global errors. Thus, the proposed multi-fidelity/multi-objective EGO can be widely applied to real-world problems.

Further, the proposed multi-fidelity/multi-objective EGO was applied to an aerodynamic airfoil shape optimization problem with two objectives: minimize $C_{\mathrm{d}}$ at cruising speed and maximize the thickness around the trialing edge. To evaluate the aerodynamic performance, XFOIL was used to construct a low-fidelity/low-cost dataset, and a Navier-Stokes solver was used to construct a high-fidelity/high-cost dataset. The results of the proposed multi-fidelity/multi-objective EGO were compared with those of the single-fidelity/multi-objective EGO. The optimization results showed that the proposed multi-fidelity/multi-objective EGO achieves greater diversity of the non-dominated solutions than the single-fidelity/multi-objective EGO. In addition, the cross-validation results showed that the proposed multi-fidelity/multi-objective EGO has fewer global errors. Finally, the proposed multi-fidelity /multi-objective EGO was applied to an aerodynamic airfoil shape optimization problem with three objectives: minimize $C_{\mathrm{d}}$ at cruising speed, maximize the thickness around the trialing edge and maximize $C_{1}$ in the landing condition. The results showed that the proposed multi-fidelity/multi-objective EGO achieves greater diversity of the non-dominated solutions than the single-fidelity/multi-objective EGO. In addition, the error between the exact value and the predicted value of the hybrid surrogate model was smaller than that of the single-fidelity model. These results 
suggest that the multi-fidelity/multi-objective EGO is suitable for real-world multi-objective design problems. In this study, we limited the optimization to two/three objectives for simple aerodynamic design problems. In the future, we expect that our algorithm will be used to solve more complicated design problems, and we will investigate the effects of the application of multi-fidelity data in advanced kriging methods such as universal kriging and anisotropy kriging.

Acknowledgments: The author would like to express deep appreciation and to special thanks to the studies funded by the Asian Human Resources Fund (AHRF) from the Tokyo Metropolitan Government to the Tokyo Metropolitan University (TMU).

Author Contributions: Atthaphon Ariyarit developed the algorithm/performed the experiment/analysis of the data and wrote the paper; Masahiro Kanazaki performed analysis of data and wrote the paper.

Conflicts of Interest: The authors declare no conflict of interest.

\section{References}

1. Wickramasinghe, U.K.; Carrese, R.; Li, X. Designing airfoils using a reference point based evolutionary many-objective particle swarm optimization algorithm. IEEE Congr. Evolut. Comput. 2010, 10, 142-149.

2. Ariyarit, A.; Kanazaki, M. Multi-modal distribution crossover method based on two crossing segments bounded by selected parents applied to multi-objective design optimization. J. Mech. Sci. Technol. 2015, 29, 1443-1448.

3. Choi, S.; Alonso, J.J.; Kroo, I.M. Two-level multifidelity design optimization studies for supersonic jets. J. Aircr. 2009, 46, 776-790.

4. Liu, W.-F.; Li, S.-Y.; Gong, Z.B.; Jiang, Z. Airfoil Optimization Design by Panel Methods for Small Size Aerial Vehicle at Low Reynolds Number. In Proceedings of the 1st International Conference on Mechanical Engineering and Material Science, Shanghai, China, 28-30 December 2012.

5. Jameson, A. Optimum aerodynamic design using CFD and control theory. AIAA Paper 1995, 1729, 124-131.

6. Choi, S.; Alonso, J.J.; Kroo, I.M.; Wintzer, M. Multifidelity design optimization of low-boom supersonic jets. J. Aircr. 2008, 46, 106-118.

7. Forrester, A.I.J.; Sobester, A.; Keane, A.J. Multi-fidelity optimization via surrogate modelling. Proc. R. Soc. Lond. A 2007, 463, doi:10.1098/rspa.2007.1900.

8. Huang, L.; Gao, Z.; Zhang, D. Research on multi-fidelity aerodynamic optimization methods. Chin. J. Aeronaut. 2013, 26, 279-286.

9. Khuri, A.I.; Mukhopadhyay, S. Response surface methodology. Wiley Interdiscip. Rev. 2010, 2, 128-149.

10. Rethore, P.E.; Fuglsang, P.; Larsen, G.C.; Buhl, T.; Larsen, T.J.; Madsen, H.A. TopFarm: Multi-fidelity optimization of offshore wind farm. In proceedings of the Twenty-first International Offshore and Polar Engineering Conference, Maui, Hawaii, USA, 19-24 June 2011.

11. Rethore, P.E.; Fuglsang, P.; Larsen, G.C.; Buhl, T.; Larsen, T.J.; Madsen, H.A. TopFarm: Multi-fidelity optimization of offshore wind farm. Wind Energy 2014, 17, 1797-1816.

12. Leon, E.R.; Le Pape, A.; Désidéri, J.A.; Alfano, D. Multi-Fidelity Concurrent Aerodynamic Optimization of Rotor Blades in Hover and Forward Flight. In Proceedings of the 40th European Rotorcraft Forum, Southampton, UK, 2-5 September 2014.

13. Leusink, D.; Alfano D.; Cinnella, P. Multi-fidelity optimization strategy for the industrial aerodynamic design of helicopter rotor blades. Aerosp. Sci. Technol. 2015, 42, 136-147.

14. Collins, K.B.; Sankar, L.N.; Mavris, D.N. Application of low-and high-fidelity simulation tools to helicopter rotor blade optimization J. Am. Helicopter Soc. 2013, 58, 1-10.

15. Fusi, F.; Guardone, A.; Quaranta, G.; Congedo, P.M. Multifidelity Physics-Based Method for Robust Optimization Applied to a Hovering Rotor Airfoil AIAA J. 2015, doi:10.2514/1.J053952.

16. Jones, D.R.; Schonlau, M.; Welch, W.J. Efficient global optimization of expensive black-box functions. J. Glob. Optim. 1998, 13, 455-492.

17. Couckuyt, I.; Deschrijver, D.; Dhaene, T. Fast calculation of multiobjective probability of improvement and expected improvement criteria for Pareto optimization. J. Glob. Optim. 2014, 60, 575-594.

18. Hupkens, I.; Emmerich, M.; Deutz, A. Faster Computation of Expected Hypervolume Improvement. arXiv 2014, arXiv:1408.7114. 
19. Orr, M.J.L. Introduction to Radial Basis Function Networks; Institute for Adaptive and Neural Computation, Edinburgh University: Edinburgh, UK, 1996.

20. Praveen, C.; Duvigneau, R. Radial basis functions and kriging metamodels for aerodynamic optimization. RapiTech: Shanghai, China, 2007; Volume 159.

21. Matheron, G. Principles of geostatistics. Econ. Geol. 1963, 58, 1246-1266.

22. Moore, R. Geostatistics in Hydrology: Kriging Interpolation; Technical Report; Mathematics Department, Macquarie University: Sydney, Australia, 1999.

23. Goldberg, D.E.; Holland, J.H. Genetic algorithms and machine learning. Mach. Learn. 1988, 3, 95-99.

24. Bader, J.; Zitzler, E. HypE: An algorithm for fast hypervolume-based many-objective optimization. Evolut. Comput. 2011, 19, 45-76.

25. Everson, R.M.; Fieldsend, J.E.; Singh, S. Full elite sets for multi-objective optimisation. In Adaptive Computing in Design and Manufacture V; Springer: London, UK, 2002; pp. 343-354.

26. Eshelman, L.J. Real-Coded Genetic Algorithms and Interval-Schemata. In Foundations of Genetic Algorithms; Elsevier: Amsterdam, The Netherlands, 1993; pp. 187-202.

27. Melanie, M. The title of the cited contribution. In An Introduction to Genetic Algorithms; Publishing House: Cambridge, MA, USA, 1999; pp. 62-75.

28. Huband, S.; Hingston, P.; Barone, L.; While, L. A review of multiobjective test problems and a scalable test problem toolkit. IEEE Trans. Evol. Comput. 2006, 10, 477-506.

29. Liem, R.P.; Mader, C.A.; Martins, J.R.R.A. Surrogate models and mixtures of experts in aerodynamic performance prediction for aircraft mission analysis. Aerosp. Sci. Technol. 2015, 43, 126-151.

30. Meckesheimer, M.; Booker, A.J.; Barton, R.R.; Simpson, T.W. Computationally inexpensive metamodel assessment strategies. AIAA J. 2002, 40.10, 2053-2060.

31. Obayashi, S.; Guruswamy, G.P. Convergence acceleration of a Navier-Stokes solver for efficient static aeroelastic computations. AIAA J. 1995, 33, 1134-1141.

32. Yoon, S.; Jameson, A. Lower-upper symmetric-Gauss-Seidel method for the Euler and Navier-Stokes equations. AIAA J. 1988, 26, 1025-1026.

33. Fujii, K.; Obayashi, S. High-resolution upwind scheme for vortical-flow simulations. J. Aircr. 1989, 26, 1123-1129.

34. Baldwin, B.S.; Barth, T.J. A One-Equation Turbulence Transport Model for High Reynolds Number Wall-Bounded Flows; NASA: Washington, DC, USA, 1990, doi:10.2514/6.1991-610.

35. Drela, M. XFOIL: An analysis and design system for low Reynolds number airfoils. In Low Reynolds Number Aerodynamics; Springer: Berlin/Heidelberg, Germany, 1989; pp. 1-12.

36. Kulfan, B.M. Universal parametric geometry representation method. J. Aircr. 2008, 45, 142-158.

37. Lorentz, G.G. Bernstein Polynomials; American Mathematical Society: Providence, RI, USA, 2012. 